\title{
Closed-loop Control of a Vibrant Duct Gravimetric Feeder
}

\author{
A. Cologni, D. Belloli, M.Madaschi, F. Previdi, Member, IEEE, S. M. Savaresi, Member, IEEE, D. Cazzola
}

\begin{abstract}
In this work the control system for a gravimetric feeder with vibrant duct for polymer extrusion process has been designed. The plant considered in this paper is a blending machine that mixes up to six components: each component is measured by a dedicated vibrant duct. All components are mixed directly in the chambers of the extruder screw. The mass delivered by each meter is measured by a load cell. The control objectives are: accurate mass flow estimate on the basis of the weight; accurate mass flow regulation; minimum settling time; robustness of the controller parameters against the variation of material and structure of meter.

Keywords: Control, plastic extrusion process, plastic metering.
\end{abstract}

\section{INTRODUCTION}

$\mathrm{C}$ ontinuous gravimetric blenders are key elements in the plastic extrusion process, which has a standard setup including a feeding section, a barrel and a head with a die for shaping $[5,6,10]$. In the feeding section, the solid polymer is fed into the extruder through a blender in the form of granulate, pellets or irregular small bits. Usually, from two to six different polymeric components are blended in the feeding section by means of gravimetric or volumetric systems. Then, the polymer is transported along the duct by means of a vibrator. The duct wall is equipped with a number of electric heaters which melt the polymer. The material is melted and pushed towards the die where the extruded final product is shaped and expelled. During the process, the polymer undergoes very complex thermo-mechanical transformations inducing strong changes in the physical properties of the material $[7,11,15]$. However the final product quality in extrusion is essentially characterized by a precisely-regulated volumetric flow of the polymer through the extruder $[3,4,12,14,15,17]$. This can be achieved by fine regulation of the mass flow delivered from the blender to the extruder. Traditionally, continuous metering of plastic was performed by volumetric blenders, which are not equipped to measure the plastic quantity actually delivered to the extruder.

A. Cologni, D. Belloli, M. Madaschi, F. Previdi are with the Dipartimento di Ingegneria dell'Infomazione, Università di Bergamo, via Marconi, 5, 24044 Dalmine (BG), Italy (corresponding author: Fabio Previdi; email: previdi@ unibg.it).

D. Belloli, S.M. Savaresi are with the Dipartimento di Elettronica e Informazione, Politecnico di Milano, Milano, Italy.

D. Cazzola is with Doteco spa, San Martino Spino (MO), Italy.
So, they are controlled in open loop and their performances are limited. Recently, a new generation of plastic blenders for extrusion has been conceived, which are equipped with load cell to measure the plastic weight fed into the extruder. The gravimetric blenders can provide accurate flow control by means of closed loop control [9]: they can give the chance of a considerable increase in performances, provided that an accurate mass flow estimate is computed on the basis of the weight measurements. In fact, in metering process, accuracy is an essential requirement in particular for additive components which are metered at very low flow values. Closed loop control of gravimetric blending provides many advantages with respect to volumetric blenders: metering is independent of material density variations; the frequent calibrations needed for volumetric feeders are not necessary; the increased accuracy considerably reduces the incidence of raw material costs, since each component is metered without any waste.

In this paper, the control system design problem for a gravimetric blender with vibrant duct is considered. Specifically, a flow-rate loop architecture has been used for dosage of each component. This control system has been designed on the basis of an estimate of the mass flow obtained by filtering the measure of weight. The control algorithm is based on a PID (Proportional, Integral, Derivative) structure tuned with the targets of reduce the settling time and increase the robustness of the controller parameters against the variation of material and differences in size of meters.

The paper is organized as follows. In Section II, the experimental equipment is illustrated and the control problems are defined. In Section III, the algorithm for mass flow estimation is described. In Section IV the meter control loop is described. Section V is devoted to concluding remarks.

\section{EXPERIMENTAL SETUP AND PROBLEM STATEMENT}

The mechanical layout of the continuous gravimetric feeder used in the present work is shown in figure 1, where an example of two component blender (the blue and the red one) is considered (see also Fig. 3). The device consists of 
different meters (up to six) whose operation is based on the transport of the material by vibrant ducts.

Each meter is made of three parts: a hopper, containing the material; a vibrator, composed of a DC motor that moves a variable eccentric mass and a duct connected to the vibrator necessary to transport the material in the extruder (in this paper the connection between vibrator and duct is considered stiff). Changing the position of the eccentric mass into the vibrator is possible to modify the force acting on the duct and then the material flow. The meter operation is based on the loss-in-weight measurement principle: the weight of the hopper is measured in real-time; so, it is possible to compute the flow by weight measurement differentiation.

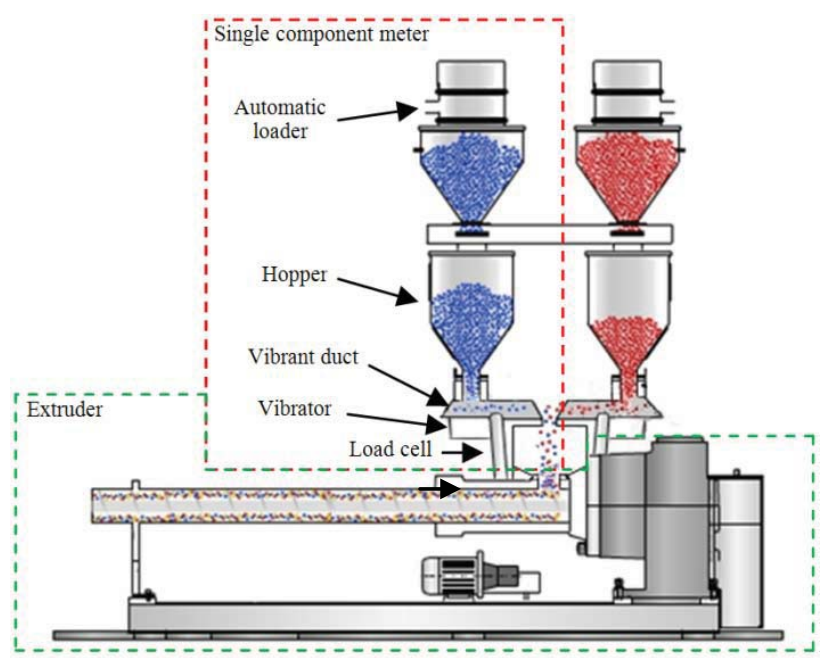

Fig. 1. Schematic of the continuous gravimetric feeder.

The machine operation is based on the following steps:

- the control of the extrusion line establishes the total reference flow of the blender with a rate of 1 sample per second;

- the total flow required is splitted to all feeder on the basis of the established recipe;

- $\quad$ each meter must ensure the delivery of the required flow-rate.

A typical work-cycle of a single meter is described in figure 2:

1. during the dosage, the material in the hopper decreases;

2. when the weight drops below a minimum threshold the pneumatic valve of the automatic loader is opened and the refill starts;

3. when the weight reaches the maximum value the pneumatic valve is set closed and the aspirator of the automatic loader is switched on;

4. after the automatic loader has been charged, the fan is turned off and the cycle can resume.
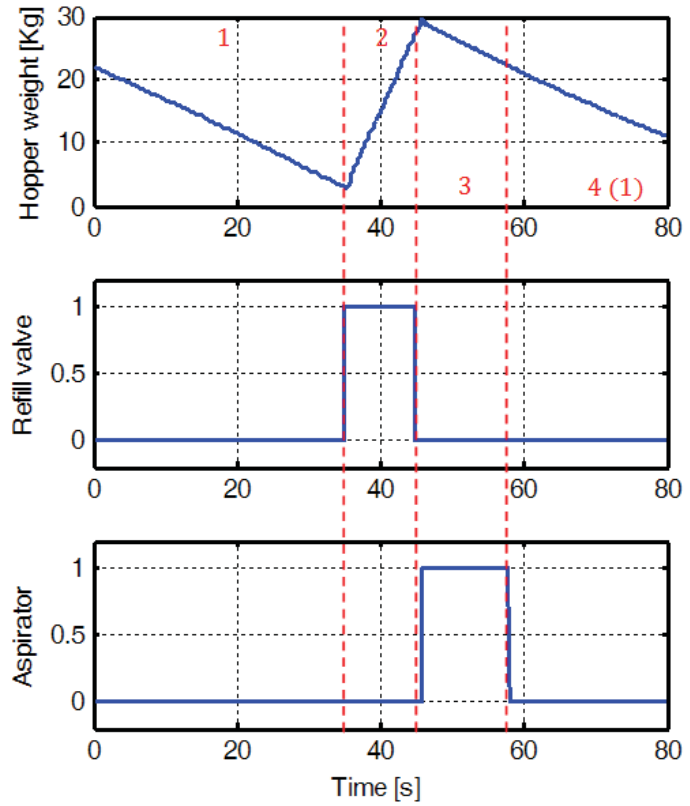

Fig. 2. Machine work cycle.

All the components dosed by the feeder are mixed directly in the extruder chambers: for this reason, an high accuracy is necessary in measuring and dosing the material, considering also that wrong dosage causes variations of pressure on the head of extruder and then low quality on the final product.

Several configurations of the meters are possible depending on the vibrators size and duct dimension, in order to satisfy a max flow rate range from $100 \mathrm{Kg} / \mathrm{h}$ to $1000 \mathrm{Kg} / \mathrm{h}$.

A typical plant uses two meters with $1000 \mathrm{Kg} / \mathrm{h}$ and two meters with $100 \mathrm{Kg} / \mathrm{h}$ : in the larger meters are usually loaded the less valuable components ("bulk" components) and in the smaller ones are charged the "additives" used in lower percentages.

The machine is regulated by a PLC, which acquires the mass measures (coming from strain gauges load cells) sampled with a 24-bit analogical input and provides the force references with a 16-bit DAC. The sample time used for the control of the feeder is $100 \mathrm{~ms}$.

A block diagram representation of the overall system is shown in figure 3 for a two components feeder. The control variables are the reference forces $\bar{f}_{1}(t)$ and $\bar{f}_{2}(t)$ of the vibrators, that operate in close loop (inside the vibrator, a current control provides proper input power). The output forces of the vibrator $f_{1}(t)$ and $f_{2}(t)$ (measured in terms of acceleration in the characterization test and called $a_{m 1}(t)$ and $a_{m 2}(t)$ ) are modulated by a sinusoid at $50 \mathrm{~Hz}$ of frequency and determine the flow-rate of material in the 
duct (which also depends from the density, structure of material and the design of the duct).

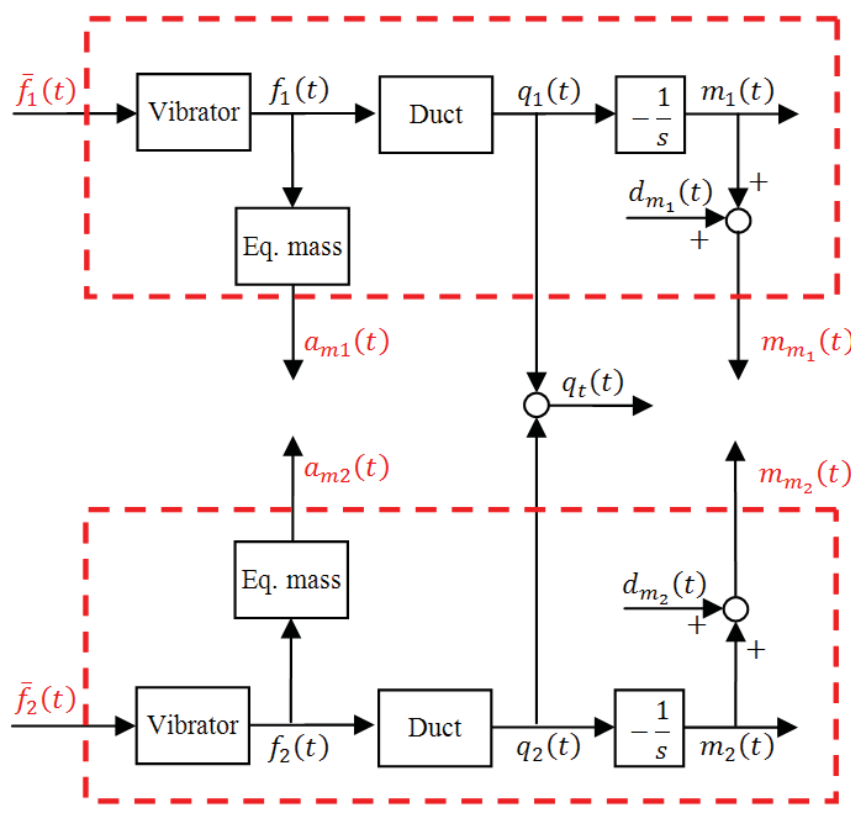

Fig. 3. System theory representation of the considered plant.

The output flow rates $q_{1}(t)$ and $q_{2}(t)$ generate a decrease of the mass in the hopper, respectively $m_{1}(t)$ and $m_{2}(t)$; the measures of mass $m_{m 1}(t)$ and $m_{m 2}(t)$ are affected by noise $d_{m_{1}}(t)$ and $d_{m_{2}}(t)$, which depend on the operating conditions (for example vibration of the floor where the feeder is installed, movement of the material inside the hopper, excitation of mechanical resonances caused by automatic loaders, etc...).

The vibrator can be described with a first order dynamic system such as:

$\frac{F(s)}{\bar{F}(s)}=\frac{\mu}{\tau \cdot s+1}$

The parameters $\mu$ and $\tau$ are dependent from the structure of the vibrator. In particular the position of the pole (represented by $\tau$ ) depends from the dynamic characteristics of the actuator and the gain (represented by $\mu$ ) depends on the spring stiffness mounted on the vibrator body.

In order to characterize this critical component some experimental test were carried out on a vibrator equipped with an accelerometer, as shown in figure 4 .

Because of the vibrator is excited with a sinusoidal signal, the measured acceleration has a component at the excitation frequency. The output force $f(t)$ is related to the acceleration amplitude, calculated as the envelope of the measured signal.

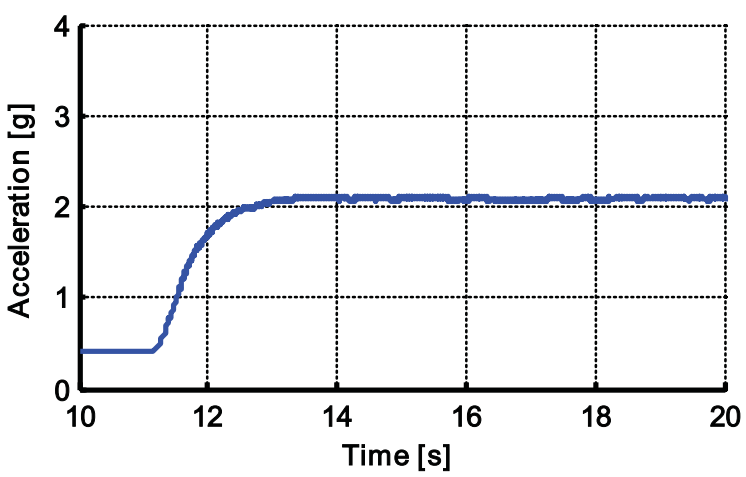

Fig. 4. Acceleration induced by the vibrator.

The other fundamental component is the duct: its characteristics determine the relation between the output force of the vibrator and the flow-rate of material exiting from the meter. The mechanical layout of this component is critical for two main reasons: it is necessary to tune the mechanical resonance of the duct in according to the excitation frequency of the vibrator (which value is $50 \mathrm{~Hz}$ in this application) and the duct design must ensure good transportation of the material. Once established the mechanical structure, it is necessary to analyze the duct behavior as a function of input force, used material and the amount of material burdens on it. In figure 5, a component with a density of $600 \mathrm{~g} / \mathrm{l}$ is analyzed.

As shown in figure, the relation between force and flow-rate is a quadratic curve: in this work the linear approximation of this function is considered in order to tune the control parameters.

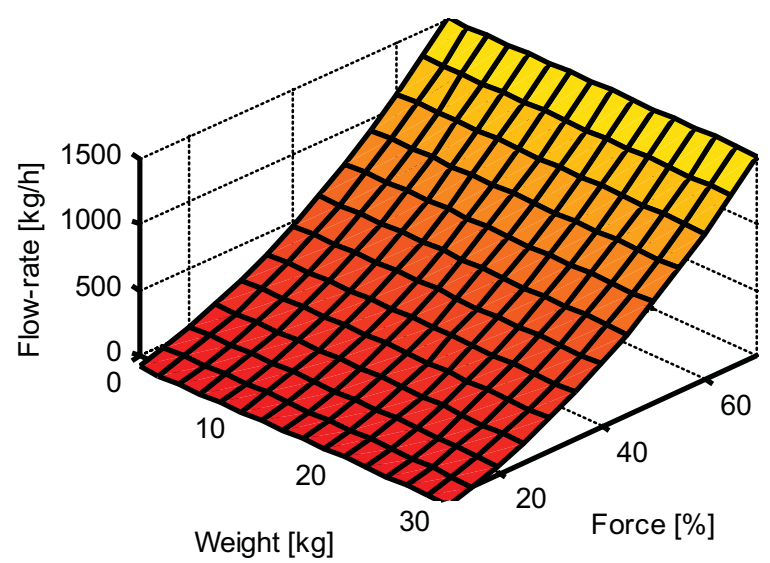

Fig. 5. Relation between flow-rate, actuation force and weight. 
For continuous blending machines, the main control objectives are:

- all the meters must deliver the exact amount of material in order to respect the desired recipe, also in transient conditions;

- the system must be able to respond to variation of flow demand as quick as possible;

- the control must be robust to variation of duct dimension and material density.

The development of the control algorithm has been performed with the feeder disconnected to the extruder, to avoid considerable plastic waste. In order to simulate the required flow variations, the flow rate reference is set directly in the machine user interface in terms of $\mathrm{Kg} / \mathrm{h}$.

Initially is necessary to obtain a flow-rate measure starting from the mass signal; then a PID control can be realized to regulate the mass flow as described in the next sections

\section{FLOW RATE ESTIMATION}

In order to control a meter, it is necessary to deduce a measure of flow-rate.

The mass flow can be theoretically calculated as the derivate of mass

$q(t)=-3600 \cdot \frac{\delta m(t)}{d t}\left[\frac{K g}{h}\right]$

where $q(t)$ is the flow-rate and $m(t)$ the mass measure.

In the discrete time domain, the derivative operation can be obtained as follow:

$\frac{\partial f(k)}{d t} \cong \frac{\Delta f(k)}{\Delta t}=\frac{f(k)-f(k-1)}{T s}$

where Ts is the sample time of the discrete signal.

This type of solution is based on the assumption of absence of noise in the input signal.

As shown in Fig. 6, the solution described above generates a flow estimation with a very low SNR (Signal to Noise Ratio); for this reason, it is necessary to use a different implementation, considering that this signal will be used for feedback.

An improvement of the mass flow signal can be obtained by reducing the amplitude of high frequency components, inserting a low-pass filter in the differentiation implementation, as shown in figure 7.
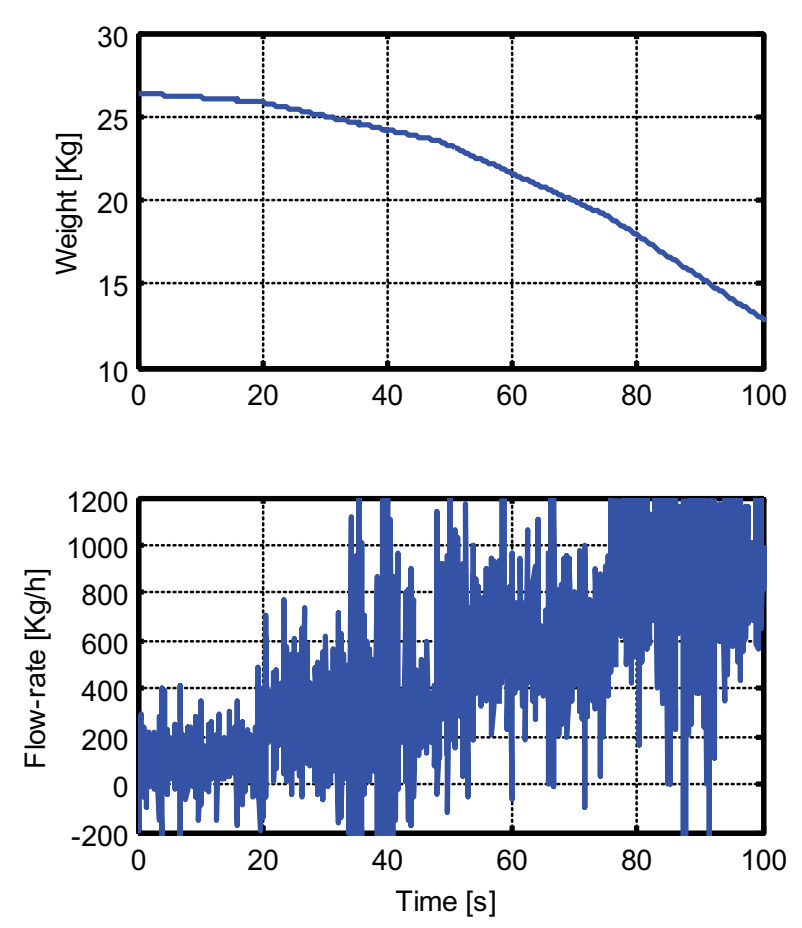

Fig. 6. Numeric derivation of mass signal.

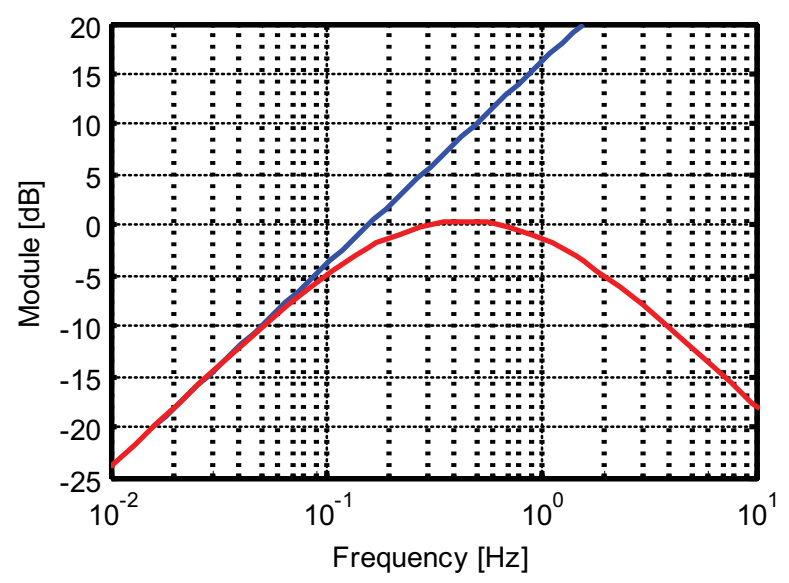

Fig. 7. Bode diagrams of the filters.

The structure of the filter is the following:

$F(s)=\frac{s}{\left(\tau_{1} \cdot s+1\right) \cdot\left(\tau_{2} \cdot s+1\right)}$

where $\tau_{1}$ and $\tau_{2}$ are the time constant of the second order filter. In this case, the parameters of the filter are $f_{1}=$ $0,2 \mathrm{~Hz}$ and $f_{2}=1 \mathrm{~Hz}$, obtaining values $\tau_{1}=0,795775 \mathrm{~s}$ and $\tau_{2}=0,159155 \mathrm{~s}$.

The implementation of the digital filter has been made using Tustin method: 
$F(z)=\frac{a_{1} \cdot z^{2}+a_{2}}{b_{1} \cdot z^{2}+b_{2} \cdot z+b_{3}}$

$a_{1}=2 \cdot \omega_{1} \cdot \omega_{2} \cdot T s$

$a_{2}=-2 \cdot \omega_{1} \cdot \omega_{2} \cdot T s$

$b_{1}=\omega_{1} \cdot \omega_{2} \cdot T s^{2}$

$b_{2}=2 \cdot \omega_{1} \cdot \omega_{2} \cdot T s^{2}-8$

$b_{3}=\omega_{1} \cdot \omega_{2} \cdot T s^{2}-2 \cdot \omega_{1} \cdot T s-2 \cdot \omega_{2} \cdot T s+4$

Applying this filter is possible to reduce the noise into the flow-rate signal as shown in Fig. 8, and use this signal to close a control loop as shown below.

A possible alternative to the implementation of a linear filter is a state variable estimation such as Kalman Filter $[1,2]$. This solution is more accurate but presents an high computational load [13]: for this reason the Kalman Filter cannot be implemented and the linear filter has been used.
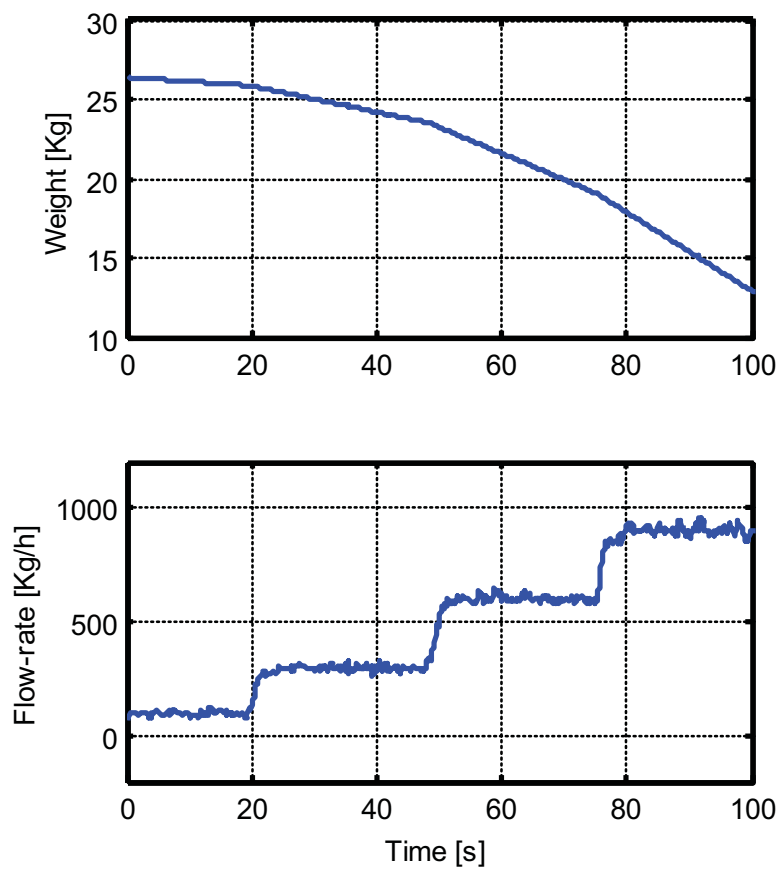

Fig. 8. Flow-rate estimation.

\section{FLOW RATE CONTROL}

After an accurate estimation of mass flow has been obtained, is possible to design the control strategy for the material flow-rate. In this work a PID controller has been used $[8,18]$. The control scheme is implemented with the addition of the anti-windup, necessary to ensure proper tracking close to the saturation zone, in particular for high flow-rate values.

The controller parameters have been tuned considering the fundamental dynamics of the system. As shown in figure 4, the response of the vibrator to a step input shows a transient of approximately $3 \mathrm{~s}$ : as described in Eq. 1 it is possible to model this component as a first order system with a time constant of 0,6 s. Approximating the relation between force and flow-rate like an algebraic constant, the other fundamental dynamic is determined by the first pole of the differentiation filter, with a time constant of $0,8 \mathrm{~s}$.

The overall meter can be represented with a second order dynamic system. The controller parameters have been tuned in according to the time constants above reported.

The parameters used for the control are:

$K_{P}=0,84\left[\frac{\% \text { force }}{\mathrm{Kg} / \mathrm{h}}\right]$

$K_{I}=0,6\left[\frac{\% \text { force }}{K g / h}\right]$

$K_{D}=0,288\left[\frac{\% \text { force }}{\mathrm{Kg} / \mathrm{h}}\right]$

Applying these parameters to the controller, the meter presents a dynamic behavior shown in figure 9. For different mass flow reference values, the close loop system has different settling times and damping: considering a material with density of $600 \mathrm{~g} / \mathrm{l}$, at $300 \mathrm{Kg} / \mathrm{h}$ the settling time is about $2,3 \mathrm{~s}$ and at $900 \mathrm{Kg} / \mathrm{h}$ it is $1,6 \mathrm{~s}$.

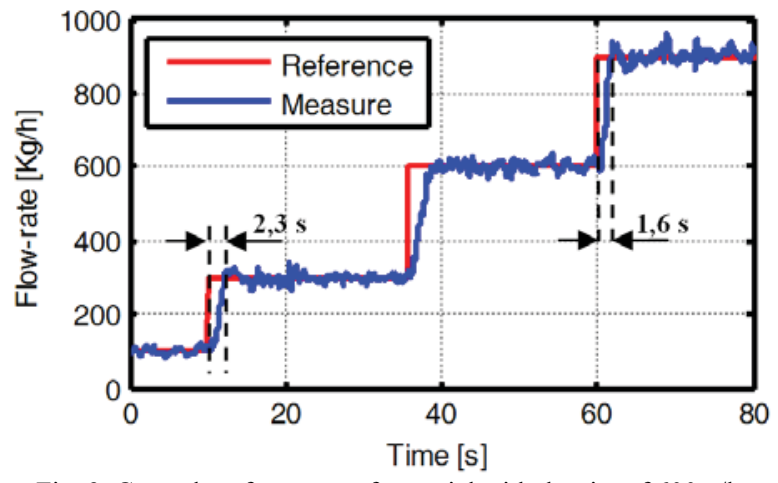

Fig. 9. Control performance of material with density of $600 \mathrm{~g} / \mathrm{l}$.

Because the system has a variable gain for different materials it is necessary to validate the control algorithm in different conditions of plastic density and form (i.e. flakes 
or granules). This test is shown in figure 10, where a material with density of $300 \mathrm{~g} / \mathrm{l}$ is used and the resulting settling time is about $4,6 \mathrm{~s}$. It is worth to note that a decrease of the material density produces an increase of the settling time of the close-loop system.

The control parameters has been tuned in order to guarantee reduced settling time in overall conditions and robustness to variations of the materials.

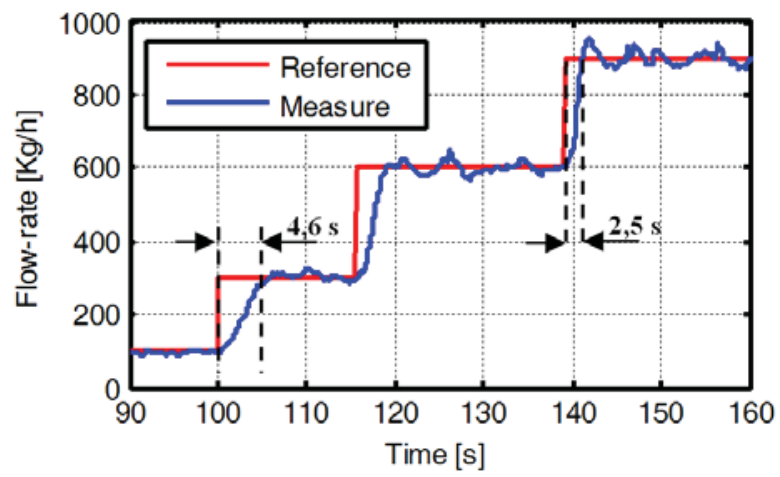

Fig. 10. Control performance of material with density of $300 \mathrm{~g} / 1$.

Finally the controlled feeder has been tested on a complete process line, where the flow mass reference is determined by the extrusion control: the resulting performances are satisfying, as shown in figure 11 .

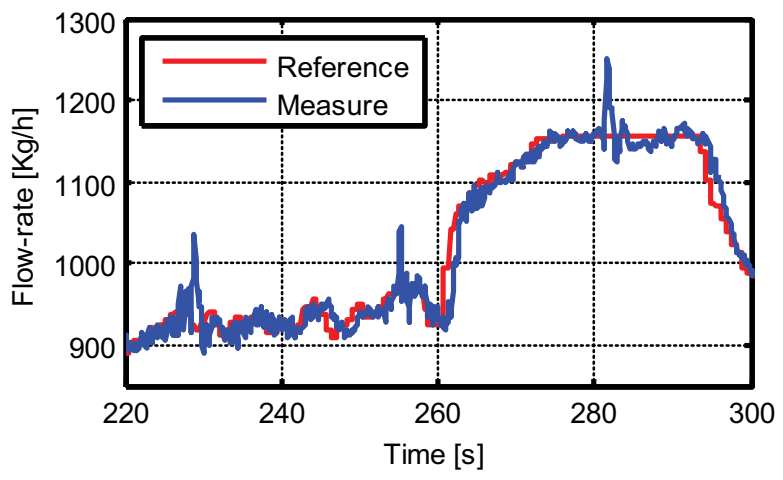

Fig. 11. Control performance on extrusion line.

\section{CONCLUDING REMARKS}

In this paper, the complete control system for a continuous gravimetric feeder with vibrant duct has been designed. In particular, the control system has been conceived to ensure efficacy with different plastic materials. The mass flow estimation has been realized applying a linear derivative filter to the mass measure acquired from the load cell. The mass flow estimation has been used for a close-loop control based on an anti-windup PID architecture.

This control has been successfully tested on different types of meters (small and big ducts) and different types of material (with variable form and density).

\section{REFERENCES}

[1] Bittanti S., G. Picci (1996). Identification, adaptation, learning : the science of learning models from data. Springer, Berlin (GER).

[2] Bittanti S., Savaresi S.M. (2000). On the parametrization and design of an Extended Kalman Filter Frequency Tracker. IEEE Transactions on Automatic Control, vol.45, n.9, pp.1718-1724.

[3] Broadhead T.O., W.I, Patterson, J.M. Dealy (1996). Closed loop viscosity control of reactve extrusion with an in-line rheometer. Polymer Engineering and Science, vol. 36, No. 23, pp. 2840-2851.

[4] Chiu S-H, S-H Pong (2000). In-line viscosity fuzzy control. Journal of Applied Polymer Science, Vol. 79, No. 7, pp. 1249-1255.

[5] Costin M.H., P.A. Taylor, J.D. Wright (1982). A critical review of dynamic modelling and control of plasticating extruders. Polymer Engineering and Science, vol. 22, No. 7, pp. 393-401

[6] Costin M.H., P.A. Taylor, J.D. Wright (1982). On the dynamics and control of a plasticating extruder. Polymer Engineering and Science, vol. 22, No. 17, pp. 1095-1106.

[7] Fenner R.T., A.P.D. Cox, D.P. Isherwood (1979). Surging in screw extruders. Polymer, vol. 20, No. 6, pp. 733-736.

[8] Guardabassi G.O., S.M. Savaresi (2001). Approximate Linearization via Feedback - an Overview. Automatica, Survey paper, vol.27, pp.1-15.

[9] Isermann R, U. Raab (1993). "Intelligent actuators - Ways to autonomous actuating systems". Automatica, vol. 29, N. 5, pp. 1315-1331.

[10] Kochhar A.K., J. Parnaby (1977). Dynamical modelling and control of plastics extrusion processes. Automatica, vol.13, pp. 177-183.

[11] Mudalamane R., D.I. Bigio (2003). Process variations and the transient behavior of extruders. AIChE Journal, vol.49, n. 12 , pp. 3150-3160.

[12] Noriega E. M. del Pilar, Rauwendaal C. (2001) Troubleshooting the extrusion process. Hanser, Munich.

[13] Previdi F., M. Lovera (2003). Identification of a class of nonlinearly time-varying models. International Journal of Adaptive Control and Signal Processing, Vol. 17, pp 3350.

[14] Previdi F., S.M. Savaresi, A. Panarotto (2006). Design of a feedback control system for real time control of flow in a single screw extruder. Control Engineering Practice, vol. 14, pp. 1111-1121.

[15] Rauwendaal C. (2001) Polymer Extrusion. Hanser, Munich.

[16] Savaresi S.M., R. Bitmead, W. Dunstan (2001). Nonlinear system identification using closed-loop data with no external excitation: the case of a lean combustion process. International Journal of Control, vol.74, pp.1796-1806.

[17] Wellstead P.E., W.P. Health, A.P. Kjaer (1998) Identification and control of web processes: polymer film extrusion. Control Engineering Practice, vol. 6, pp. 321331.

[18] Astrom K.J., T. Hagglund. (2000). PID controllers: theory design and tuning. Springer-Verlag, Berlin (GER). 